\title{
Improved Multi-Sensor Integrated Navigation System for Land-Vehicles
}

\author{
Zhang Yuyang, Cai Bogen, Wang Jian
}

\author{
School of Electronic and Information Engineering, Beijing Jiaotong University, Beijing 100044, \\ China; \\ edwin01@163.com
}

Keywords: extended Kalman filter (EKF), inertia system, integrated navigation system

\begin{abstract}
Standalone Beidou satellite navigation system cannot achieve the high positioning accuracy when the signal is obscured. An improved filtering algorithm based on Extended Kalman filter (EKF) is proposed after comparing the advantages and disadvantages of the filtering method. Navigation parameters from SINS and platform error angles are chosen as the state of the system, and navigation parameters from passive-BD are chosen as measurements. The final optimal estimation of navigation parameters including position and speed can be calculated directly. The experimental result indicates that the new algorithm can effectively solve the nonlinearity problem of state equations, and provide continuous positioning result.
\end{abstract}

\section{Introduction}

Beidou navigation system developed by China is a navigation and positioning system that can provide quick positioning and timing services. But in the urban areas, Beidou accuracy will deteriorate due to the multipath effects and signal degradation. Inertial Navigation System (INS) is a self-contained accurate high-rate system that can provide linear acceleration and angular rate measurements. INS has a good concealment and high update rate, as a matter of fact, it can be used as an auxiliary navigation system when Beidou is outage. Though INS is accurate in the short-term, its errors will accumulate due to measurement integration and can grow without bounds. In fact, this is the major limitations of Inertial Navigation System. Both Beidou and INS have advantages and disadvantages, fortunately, their disadvantages are complementary. Beidou has a good long-term stability while INS is accurate in the short-term with high output rate. That is the base of the BD/INS integrated navigation system.

There are two basic ways to achieve integrated navigation system, one is loop feedback method and the other is the optimal estimation. Because of the randomness in subsystems and measurement error, the optimal estimation is consider better than the loop feedback method. Kalman filter is the most commonly used method in optimal estimation. Although the Kalman filter is widespread used in engineering field, it can only use to process the linear system, and we usually use the non-linear model for land vehicles. In this paper, the Extended Kalman Filter is introduce to fit with the non-linear model of BD/INS integrated navigation system. The experimental result indicate that the $\mathrm{BD} / \mathrm{INS}$ integrated navigation system based on EKF can maintain a good positioning accuracy while Beidou is outage. Not only is the system improve the accuracy, but also the reliability.

\section{Inertial Navigation System}

System structure. The inertial sensors used in this system is a low-cost, three-dimensional multi-sensor system which consist of a three-axes accelerometer and a three-axes gyroscope. The Y axis of both the accelerometer and the gyroscope is pointing towards the nose of the vehicle, also known as the nominal direction of travel. The $\mathrm{X}$ axis is orthogonal to $\mathrm{Y}$ axis pointing towards the starboard side of the vehicle, while the $\mathrm{Z}$ axis is nominally pointing up. A wheel speed sensor is also arrange to the system to pursue the vehicle speed. The arrangement of these sensors with respect to the body frame is depicted in Fig. 1. 


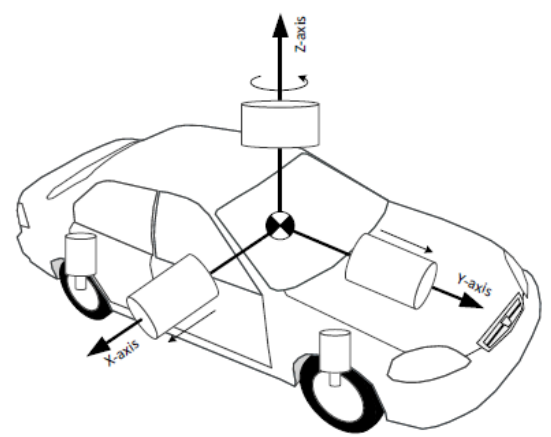

Fig. 1 Arrangement of inertial sensor system sensors

Mechanical layout. The purpose of calculating Mechanical layout is to achieve the position of the vehicle. In particular, use the output of the Gyroscope, calculate the attitude. Then use the attitude to convert force in body frame into ECEI frame. By integrate twice, we can get the position in the current time. A block diagram that illustrates the mechanization system is shown in Fig. 2.

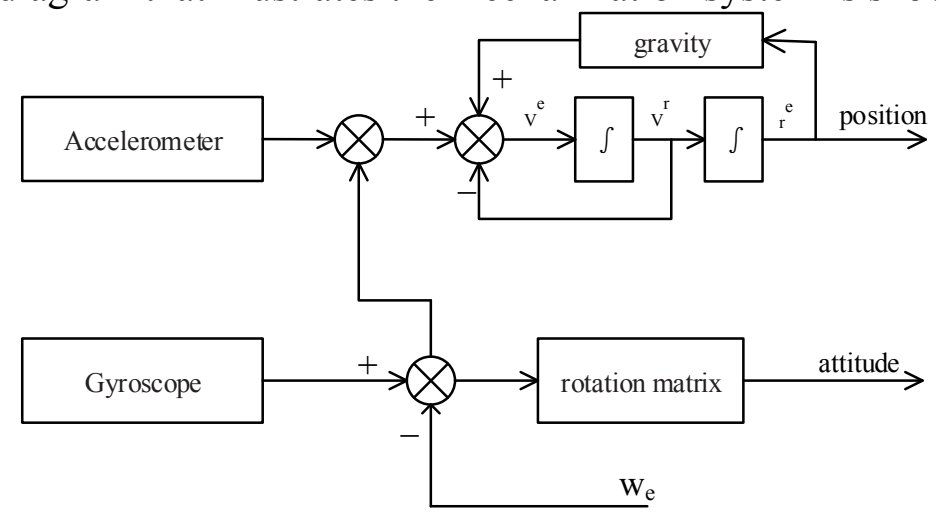

Fig. 2 mechanization block diagram

To get the change of position and velocity, we should first calculate the quaternion vector form IMU output.

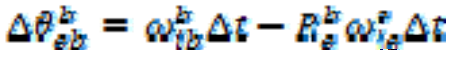

$$
\begin{aligned}
& |\theta|^{2}=\theta_{x}^{2}+\theta_{y}^{2}+\theta_{z}^{2} \\
& Q_{k+1}=Q_{k}+[(\cos \theta / 2-1) I+1 / \theta * \sin \theta / 2 * S] Q_{k}
\end{aligned}
$$

Where $\omega_{t z}^{b}$ is the output of the gyroscope, $s=\Omega \Delta t$. Through the updated quaternion vector, we can calculate the velocity.

$$
\begin{aligned}
& \Delta V_{f}^{z}=\int_{v_{\mathrm{R}}+4}^{v_{\mathrm{R}}+4} R_{f}^{b} f^{b} d t \\
& =R_{F}^{b}\left(t_{k}\right)\left[\Delta V_{f}^{b}+\Omega_{\mathrm{ez}}^{b} \frac{\left(t_{\mathrm{F}+2}-t_{\mathrm{F}}\right)}{2} \Delta V_{f}^{b}\right] \\
& =R_{v}^{L}\left(t_{k}\right)\left[E+\frac{1}{z} S^{b}\right] \Delta V_{f}^{b} \\
& V_{k+1}^{e}=V_{k}^{e}+\frac{1}{2}\left(\Delta V_{k}^{e}+\Delta V_{k+1}^{e}\right)
\end{aligned}
$$

The vehicle's position is obtained from its velocity depicted by Equation (5). And after some mathematically manipulation, we get the following form:

$$
r_{k+1}^{e}=r_{k}^{e}+0.5\left(V_{k+1}^{e}+V_{k}^{e}\right) \Delta t
$$

\section{EKF-Based BD/INS Integration Algorithms}

To mitigate errors of the Beidou navigation system, the INS which has complementary characteristics to Beidou can be integrated with it using estimation algorithms. The estimation 
algorithm in this paper is based on extended Kalman filter (EKF). This integration can achieve a superior accuracy compared to what any of the other standalone systems can provide. There are two main kind of Kalman filter that are usually work for BD/INS integration system, they are classic Kalman filter and extended Kalman filter. The classic Kalman filter can only deal with linearized model system, whereas EKF can take care of both. The EKF can keep errors within the acceptable linearization range by linearizing the system around the corrected state. A general block diagram of the $\mathrm{BD} / \mathrm{INS}$ integrated system is shown in fig. 3 .

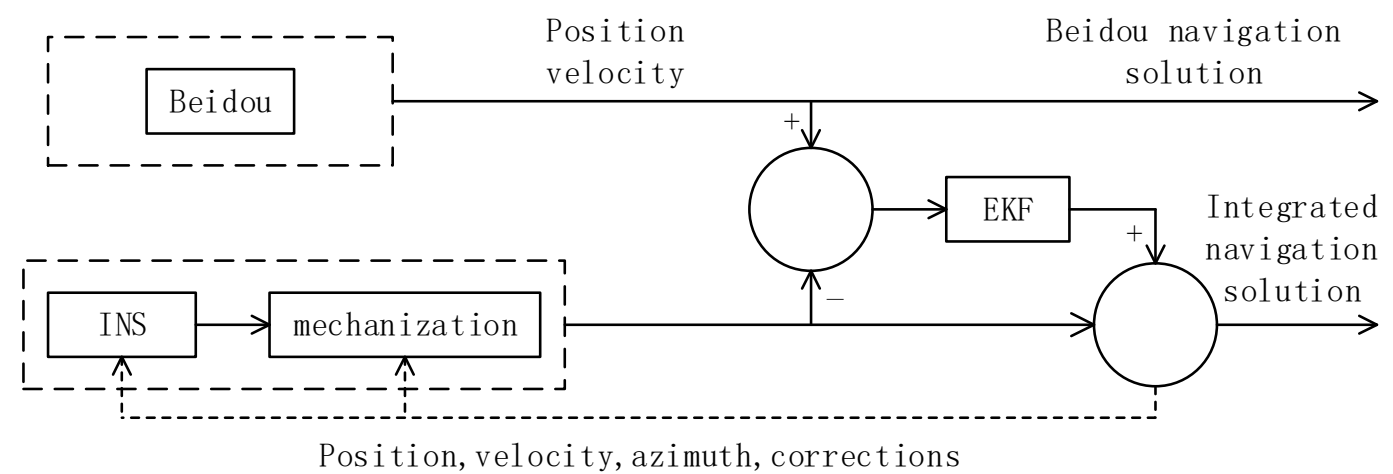

Fig. 3 BD/INS integrated system

The system model derived by linearization of the INS mechanization equations.

$$
X^{\prime}(t)=\left[\begin{array}{c}
\delta r^{t} \\
\delta v^{t} \\
\delta a^{t} \\
d \\
b
\end{array}\right]=\left[\begin{array}{c}
\delta V^{t} \\
-F^{t} a^{t}+N^{t} \delta r^{t}+R_{b-}^{t} b \\
R_{b}^{t} d \\
-A d+W W_{d} \\
-B b+b V_{k}
\end{array}\right]
$$

\section{Experimental Work and Results}

A real road experiment is proceed to examine the performance of the proposed improved multi-sensor integrated navigation system using ADI16488 MEMS IMU along with UB370 Beidou receiver. Fig. 4,5 shows the equipment inside a car that was used to conduct the road experiments to collect the data. A precision RTK is using as the reference systems.

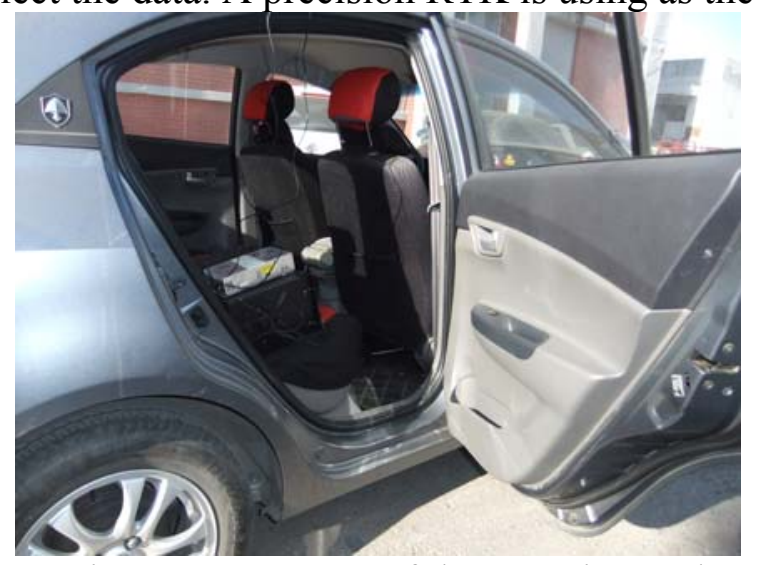

Fig. 4 Appearance of the experimental car

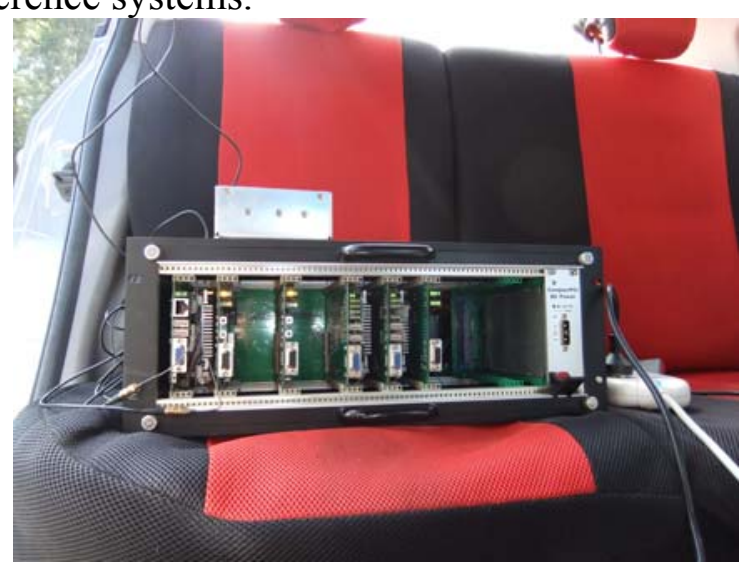

Fig. 5 Equipment for data collection

The experimental was conducted in the highway with a tunnel, where the Beidou signal is block. The outage duration was about $60 \mathrm{~s}$. The response and accuracy of the algorithm was analyzed. 

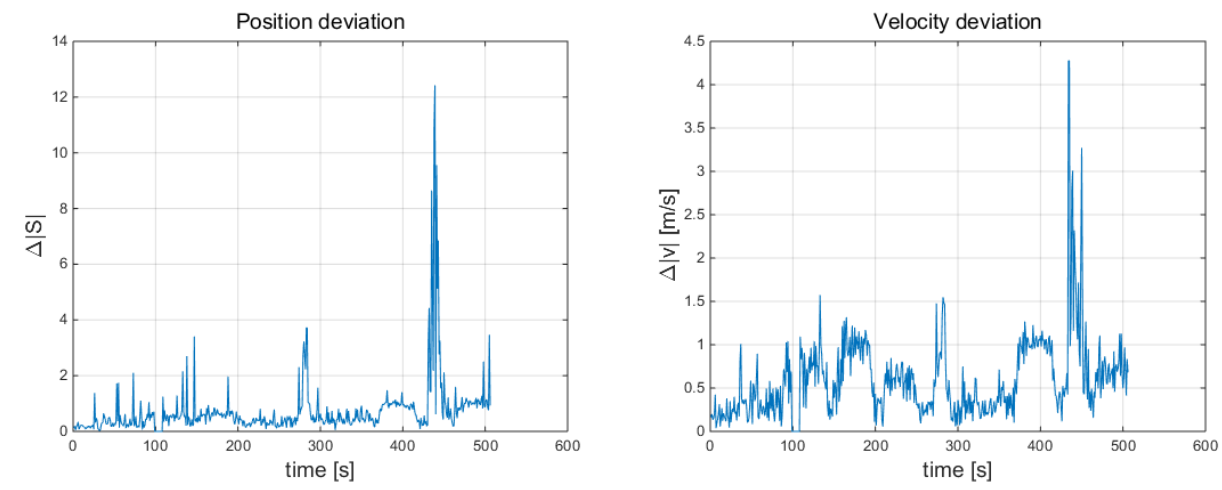

Fig. 6 Position, velocity deviation

The summarizes of the RMS errors during the outages is show in Table 1.

Table 1 RMS errors during 60 -second outages

\begin{tabular}{|c|c|c|c|c|c|c|c|c|c|}
\hline \multirow{2}{*}{ Time } & \multicolumn{3}{|c|}{ Position(m) } & \multicolumn{3}{|c|}{$\operatorname{Velocity}(\mathrm{m} / \mathrm{s})$} & \multicolumn{3}{|c|}{$\operatorname{Attitude}\left({ }^{\circ}\right)$} \\
\hline & $\mathrm{x}$ & $\mathrm{y}$ & $\mathrm{z}$ & $\mathrm{x}$ & $\mathrm{y}$ & $\mathrm{z}$ & Roll & Pitch & Azi \\
\hline 10 & 0.96 & 0.06 & 0.17 & 0.58 & 0.01 & 0.48 & 0.89 & 0.34 & 0.90 \\
\hline 20 & 2.45 & 0.89 & 1.54 & 1.05 & 0.18 & 0.87 & 1.73 & 0.55 & 1.48 \\
\hline 40 & 6.31 & 3.53 & 4.32 & 1.91 & 0.43 & 1.36 & 1.86 & 1.35 & 1.97 \\
\hline 60 & 9.23 & 4.53 & 5.59 & 3.61 & 0.83 & 2.19 & 2.31 & 1.58 & 2.80 \\
\hline
\end{tabular}

It can be observed from the experimental results that after 60-second outages of Beidou signal, the position and velocity errors are still within control, and we can get a continuous and reliable position of the vehicle.

\section{Summary}

This paper introduced a BD/INS integrated system which based on EKF that can continuously keep the linearization within an acceptable, accurate range. Road test results showed that the proposed system can keep the errors bounded while the Beidou is outages.

\section{References}

[1] Deepak Bhatt, Priyanka Aggarwal, A novel hybrid fusion algorithm to bridge the period of GPS outages using low-cost INS, Expert Systems with Applications. 41 (2014) 2166-2173.

[2] Tashfeen B. Karamat, Mohamed M. Atia, An Enhanced Error Model for EKF-Based Tightly-Coupled Integration of GPS and Land Vehicle's Motion Sensors, Sensors 2015, 15, 24269-24296;

[3] WangJian. Researeh of the Train Positioning Technologies Based on GNSS Technology [D]. BeiJing. Beijing Jiaotong University, 2007.

[4] M. Malleswaran, V. Vaidehi, IMM-UKF-TFS Model-based Approach for Intelligent Navigation, doi:10.1017/S0373463313000404, THE JOURNAL OF NAVIGATION (2013), 66, 859-877.

[5] Zengke Li, Jian Wang, Binghao Li, GPS/INS/Odometer Integrated System Using Fuzzy Neural Network for Land Vehicle Navigation Applications. THE JOURNAL OF NAVIGATION (2014), 67, 967-983. 